\title{
Laryngotracheal stenosis in children following cardiac surgery: a retrospective review
}

\author{
Caruso G., Van Deenen D., Willems A., Van Der Linden P. \\ Dept. of Anaesthesiology \& Intensive Care, HUDERF, Université Libre de Bruxelles, Belgium
}

\section{Background and Goal of Study}

$\checkmark$ Laryngotracheal stenosis is a rare but potentially life threatening complication after surgical repair for congenital heart disease (1).

$\checkmark$ Aim of the study: to determine incidence and contributing factors of such complications in our population.

\section{Materials and Methods}

$\checkmark$ No financial disclosure, IEC approval.

$\checkmark$ Medical chart review of 2121 children undergoing cardiac surgery under cardiopulmonary bypass between January 2002 and July 2015. Twelve children developed laryngotracheal stenosis : glottic stenosis $(\mathrm{N}=2)$, subglottic stenosis $(\mathrm{N}=8)$ and tracheal stenosis $(\mathrm{N}=2)$.

$\checkmark$ These twelve children were compared to " controls » using matching with a 1:2 ratio, considering age and weight.

$\checkmark$ Recognized risk factors for laryngotracheal stenosis were compared between the two groups of patients using the Mann-Whitney U test for continuous variables and the $\mathrm{x}^{2}$-test for categorical variables.

\section{Results}

$\checkmark$ Demographic and preoperative characteristics of the studied population are presented in Table 1, perioperative and postoperative characteristics in Table 2.

Table 1. Demographic and preoperative characteristics

\begin{tabular}{|c|c|c|c|c|}
\hline \multicolumn{2}{|c|}{ Variables } & $\begin{array}{l}\text { No } \\
\text { laryngotracheal } \\
\text { stenosis }(\mathrm{N}=24)\end{array}$ & $\begin{array}{l}\text { Laryngotracheal } \\
\text { stenosis }(\mathrm{N}=12)\end{array}$ & $\begin{array}{c}\text { P- } \\
\text { value }\end{array}$ \\
\hline \multicolumn{2}{|l|}{ Gender, male } & $9(37.5 \%)$ & $6(50.0 \%)$ & 0.473 \\
\hline \multicolumn{2}{|l|}{ Age (months) } & $3.6(1.5-5.3)$ & $2.9(1.7-4.5)$ & 0.873 \\
\hline \multicolumn{2}{|c|}{ Preoperative weight $(\mathrm{kg})$} & $4.3(3.7-5.0)$ & $4.3(3.5-5.1)$ & 0.915 \\
\hline \multicolumn{2}{|c|}{ Prematurity } & $1(4.2 \%)$ & $4(33.3 \%)$ & 0.017 \\
\hline \multicolumn{2}{|c|}{ Genetic abnormalities } & $7(29.2 \%)$ & $7(58.3 \%)$ & 0.091 \\
\hline \multirow{5}{*}{$\begin{array}{l}\text { ASA } \\
\text { physical status }\end{array}$} & 1 & $0(0 \%)$ & $0(0 \%)$ & \multirow{5}{*}{0.355} \\
\hline & 2 & $0(0 \%)$ & $1(8.3 \%)$ & \\
\hline & 3 & $17(70.8 \%)$ & $8(66.7 \%)$ & \\
\hline & 4 & 7 (29.2\%) & $3(25.0 \%)$ & \\
\hline & 5 & $0(0 \%)$ & $0(0.0 \%)$ & \\
\hline \multicolumn{2}{|c|}{ Cyanotic heart disease } & $15(62.5 \%)$ & $5(45.5 \%)$ & 0.344 \\
\hline \multicolumn{2}{|l|}{ Elective surgery } & $20(83.3 \%)$ & $9(75.0 \%)$ & 0.551 \\
\hline \multicolumn{2}{|l|}{ Redo surgery } & $4(16.7 \%)$ & $2(16.7 \%)$ & 1.000 \\
\hline \multirow{6}{*}{$\begin{array}{l}\text { RACHS-1 score } \\
\text { - Risk category }\end{array}$} & 1 & $0(0 \%)$ & $0(0 \%)$ & \multirow{6}{*}{0.672} \\
\hline & 2 & $8(47.1 \%)$ & $5(41.7 \%)$ & \\
\hline & 3 & $7(41.2 \%)$ & $5(41.7 \%)$ & \\
\hline & 4 & $1(5.9 \%)$ & $2(16.7 \%)$ & \\
\hline & 5 & $0(0 \%)$ & $0(0 \%)$ & \\
\hline & 6 & $1(5.9 \%)$ & $0(0 \%)$ & \\
\hline
\end{tabular}

Table 2. Perioperative and postoperative characteristics

\begin{tabular}{|c|c|c|c|c|}
\hline \multicolumn{2}{|c|}{ Variables } & $\begin{array}{l}\text { No } \\
\text { laryngotracheal } \\
\text { stenosis }(\mathrm{N}=24)\end{array}$ & $\begin{array}{l}\text { Laryngotracheal } \\
\text { stenosis ( }=12)\end{array}$ & $\begin{array}{c}\text { P- } \\
\text { value }\end{array}$ \\
\hline \multicolumn{2}{|c|}{ Surgery time (min) } & $\begin{array}{c}222.5 \\
(194.5-280.5)\end{array}$ & $\begin{array}{c}218.0 \\
(179.0-298.0)\end{array}$ & 0.763 \\
\hline \multicolumn{2}{|l|}{ CPB time (min) } & $\begin{array}{c}114.0 \\
(83.0-148.0)\end{array}$ & $\begin{array}{c}125.0 \\
(71.0-148.0)\end{array}$ & 0.606 \\
\hline \multicolumn{2}{|c|}{ Aortic cross clamp } & 22 (95.7\%) & $11(91.7 \%)$ & 0.630 \\
\hline \multicolumn{2}{|c|}{$\begin{array}{l}\text { Aortic cross clamp } \\
\text { time (min) }\end{array}$} & $49.0(37.0-75.0)$ & $68.5(37.0-75.0)$ & 0.450 \\
\hline \multicolumn{2}{|c|}{ Circulatory arrest } & $2(8.3 \%)$ & $2(16.7 \%)$ & 0.453 \\
\hline \multirow{3}{*}{$\begin{array}{l}\text { Cormack } \\
\text { Score }\end{array}$} & 1 & 17 (85.0\%) & $4(66.7 \%)$ & \multirow{3}{*}{0.171} \\
\hline & 2 & 3 (15.0\%) & $1(16.7 \%)$ & \\
\hline & 3 & $0(0 \%)$ & $1(16.7 \%)$ & \\
\hline \multirow{4}{*}{$\begin{array}{l}\text { Endo- } \\
\text { tracheal tube } \\
\text { size }(\mathrm{mm})\end{array}$} & 3,0 & $3(13,6 \%)$ & $3(3,33 \%)$ & \multirow{4}{*}{0.038} \\
\hline & 3,5 & $13(59,1 \%)$ & $1(1,11 \%)$ & \\
\hline & 4,0 & $4(18.2 \%)$ & $5(55.6 \%)$ & \\
\hline & 4,5 & 2 (9.1\%) & $0(0 \%)$ & \\
\hline \multirow{4}{*}{$\begin{array}{l}\text { Num. of } \\
\text { intubation } \\
\text { attempts }\end{array}$} & 1 & 16 (66.7\%) & $1(8.3 \%)$ & \multirow{4}{*}{0.005} \\
\hline & 2 & $6(25.0 \%)$ & $2(16.7 \%)$ & \\
\hline & 3 & $1(4.2 \%)$ & 4 (33.3\%) & \\
\hline & $\geq 4$ & $1(4.2 \%)$ & $5(41.7 \%)$ & \\
\hline \multicolumn{2}{|c|}{$\begin{array}{l}\text { First extubation } \\
\text { attempt (day) }\end{array}$} & $3.5(1.0-7.0)$ & $6.0(4.0-10.0)$ & 0.164 \\
\hline \multicolumn{2}{|c|}{$\begin{array}{l}\text { Total duration of } \\
\text { ventilation (hours) }\end{array}$} & $\begin{array}{c}72.0 \\
(22.0-125.0)\end{array}$ & $\begin{array}{c}156.5 \\
(104.0-192.0)\end{array}$ & 0.047 \\
\hline
\end{tabular}

\section{Discussion}

$\checkmark$ Incidence of laryngotracheal stenosis was very low in our population, and in accordance with the available literature (1)

$\checkmark$ Prematurity, greater endotracheal tube size and greater number of endotracheal intubation attempts were significantly associated with laryngotracheal stenosis after surgical repair for congenital heart disease.

$\checkmark$ Our findings slightly differed from those of Mossad et al., which highlighted younger age and prolonged ventilation as risk factors for subglottic stenosis after congenital hearth surgery (1). Literature on the topic is scarce.

$\checkmark$ Considering that total duration of ventilation, but not time to first extubation attempt, was different between the two groups; laryngotracheal stenosis was probably more a consequence rather than a risk factor for prolonged ventilation after surgery.

$\checkmark$ Strength of the study: matched comparison between groups for considerable number of potentially risk factors for laryngotracheal stenosis.

$\checkmark$ Limitations of the study: we were not able to evaluate whether cuffed or uncuffed endotracheal tube played a role because this information was poorly reported in the medical chart. Because of the retrospective design of our study, we couldn't determine causality relationship, but only association.

\section{Conclusion}

$\checkmark$ Prematurity, greater endotracheal tube size and greater number of endotracheal intubation attempts were significantly associated with laryngotracheal stenosis after surgical repair for congenital heart disease in our population. 